\title{
EPISTEMOLOGÍA DE VIRTUDES ROBUSTA: SOBRE LOS LÍMITES Y LAS POSIBILIDADES DE SU APLICACIÓN A LA PRUEBA DE LOS HECHOS EN EL DERECHO
}

\author{
PEDro H. HADdAD BERNAT \\ Universitat de Girona \\ pedro.haddad.bernat@gmail.com
}

RESUMEN: El objetivo del presente trabajo es definir los rasgos generales de un modelo de teoría del conocimiento viable para el ámbito probatorio judicial. En concreto, se estudian los límites y las posibilidades de aplicación al derecho de una de las propuestas más relevantes en la epistemología contemporánea: la epistemología de virtudes robusta. Como mostraré aquí, dicho proyecto es inadecuado pues un epistemología de la prueba jurídica satisfactoria y completa exige la conjunción tanto de la perspectiva del agente epistémico (el juez de los hechos) como de la perspectiva del sistema probatorio (las reglas o condiciones de indagación).

PALABRAS CLAVE: epistemología jurídica, razonamiento probatorio, teoría de la prueba, virtudes epistémicas, virtudes judiciales

SUMMARY: The purpose of this paper is to define the general features of a suitable epistemology for law. In particular, the paper is concerned with a very influential project that is nowadays offered in the literature: robust virtue epistemology. As I will show here, such a project is untenable for law, since a satisfactory and complete epistemology of legal proof requires the conjunction of both the agent's perspective (the "trier-of-facts") and the inquiry system's perspective (the rules of evidence).

KEY WORDS: legal epistemology, judicial reasoning, legal fact-finding, epistemic virtues, virtue jurisprudence

\section{Dos modelos de epistemología jurídica}

La epistemología general es la disciplina filosófica que se encarga de reflexionar sobre el conocimiento, la estructura lógica de su justificación y sus posibilidades de existencia. Se podría decir en términos muy generales que, siendo nosotros (el género humano) y el mundo los principales objetos de estudio filosófico, el conocimiento es una de las formas de conexión entre nosotros y ese mundo, y la epistemología es el estudio de las condiciones y características de dicha conexión.

La epistemología jurídica, por su parte, es una forma de epistemología aplicada. Además, si se quiere, es un capítulo dentro de la teoría de la adjudicación (parte de la teoría general del derecho) que se ocupa del estudio de la posibilidad de que las decisiones judiciales en materia de hechos sean casos de conocimiento. Hacer epistemo- 
logía jurídica consiste en aplicar al caso de la prueba judicial ${ }^{1}$ los elementos y las herramientas que la teoría del conocimiento aporta con miras, entre otras cosas, a evaluar las posibilidades y condiciones necesarias para que el resultado probatorio (la decisión en materia de hechos) sea verdadero y ostente el rango de conocimiento. ${ }^{2}$

${ }^{1}$ Con el fin de evitar posibles confusiones, cabe advertir desde ahora que a lo largo del texto usaré indistintamente expresiones como "ámbito probatorio", "derecho", "contexto jurídico" o "caso jurídico", entre otras, para referirme única y exclusivamente al ámbito de la prueba de los hechos en un proceso judicial. Debe entenderse además que tal ámbito agota la dimensión o espacio de aplicación de la epistemología general a la que llamo "epistemología jurídica".

${ }^{2}$ Una vez que hemos enfatizado la búsqueda de la verdad o el conocimiento de los hechos en el ámbito probatorio, conviene hacer una serie de aclaraciones con el objetivo de hacer explícitos los presupuestos teóricos subyacentes en este artículo. La primera cuestión es distinguir entre los fines del proceso y los fines de la prueba (entendiendo "prueba" como actividad). Si bien podría quedar a discusión que el objetivo primario del proceso sea la averiguación de la verdad — cosa que yo estaría dispuesto a poner en tela de juicio-, no puede dudarse, en cambio, de que el fin de la prueba sea otro que averiguar la verdad sobre los hechos aducidos por las partes en un proceso. En efecto, ¿qué sentido tendría la actividad probatoria misma como parte del gran esquema del proceso si no fuera intentar demostrar la verdad de los hechos? La acción misma de presentar pruebas en el marco de un proceso judicial es absurda si no se asume que el objetivo es demostrar la verdad de determinada serie de enunciados sobre hechos.

Una segunda cuestión — que puede parecer trivial, pero que de no explicitarse puede conducir a inútiles disputas verbales - es relativa a la necesidad de distinguir entre afirmar que la averiguación de la verdad es imposible en el proceso y afirmar que es difícil. Algunos sistemas probatorios (entendidos como marcos de reglas de indagación) tienden a hacer esa labor más fácil, otros tienden a hacerla más difícil, pero lo importante es entender que no es lo mismo que la tarea de probar sea difícil — mas en principio posible - a que no se pueda lograr.

En consonancia con lo anterior, preguntarnos si un sistema $X$ tiende a facilitar el descubrimiento de la verdad o a dificultarlo es un problema empírico del que no me ocuparé aquí. Si al lector le interesa cómo se puede abordar este problema, remítase a Laudan 2016. Para los efectos de este trabajo, basta reconocer que hay una multiplicidad de sistemas probatorios (actuales y posibles) con tendencias y diseños muy variados. Hay más de una manera legítima de construir un sistema de indagación y en la base de los diseños que se eligen se encuentran la visión, las prioridades y los valores de la comunidad epistémica que lo construye.

Por último, es importante tener presente en todo momento que en este trabajo no se presume en modo alguno que probar en el contexto de un proceso judicial sea algo que irrevocablemente implique la verdad de lo probado (como si la prueba de que $p$ estuviera atada conceptualmente a la verdad de $p$ ). Reconozco y defiendo que una cosa es "probar jurídicamente que $p$ " y otra cosa es afirmar que " $p$ es verdad". En el marco de un proceso judicial es perfectamente posible probar proposiciones sobre hechos que sean falsas. Esto significa que el vínculo entre prueba y verdad es más de orden teleológico que conceptual. Sobre esto, véase Ferrer Beltrán 2005. 
El objetivo de este trabajo es determinar cuáles son las características o rasgos que un modelo de epistemología jurídica viable debe poseer. Para ello tendremos en mente dos perspectivas o modelos abstractos: el modelo de la epistemología centrada en el agente (ECA, en adelante) y el modelo más tradicional de la epistemología centrada en el sistema (ECS, en adelante). Ambos modelos o perspectivas son formas de hacer epistemología del derecho que se distinguen por aquello a lo que tienden a dar preponderancia al momento de abordar las posibilidades del conocimiento de los hechos que dieron vida a un litigio.

La ECS, como su nombre lo indica, tiende a centrarse en el estudio de las características del sistema probatorio. Es una perspectiva desde la cual el cúmulo de reglas probatorias se concibe como un "sistema de indagación" que se somete a escrutinio mediante la aplicación de las herramientas que proporciona la teoría del conocimiento entendida en sentido lato, entre otras: la teoría de la probabilidad, las concepciones de la inducción y la abducción, las teorías de la estructura lógica de la justificación epistémica (coherentismo/fundacionismo), los grados de confirmación, el análisis tripartito del conocimiento. Así pues, la ECS consiste básicamente en la aplicación de la epistemología al estudio y la evaluación del sistema probatorio en todo lo relativo a reglas probatorias epistémicas y contraepistémicas, reglas de exclusión, criterios de admisión, definición y calificación de estándares probatorios, reglas de carga de la prueba, entre otros.

La forma más tradicional o habitual en la literatura sobre epistemología aplicada al derecho es, sin duda, la ECS. Tal vez el caso más claro de modelo epistemológico con esta visión sea la obra de Larry Laudan (2008 y 2016). Otro gran ejemplo es el caso de Michele Taruffo (1992). ${ }^{3}$ Las reflexiones de estos autores son ejemplos paradigmáticos de cómo aplicar las herramientas de la teoría del conocimiento al análisis, la crítica y la depuración de los sistemas probatorios en el derecho.

En cambio, la ECA es un modelo epistemológico en el que el enfoque del análisis se dirige no tanto al sistema, sus reglas, estándares y criterios, como al agente o los agentes que operan dentro del sistema.

\footnotetext{
${ }^{3}$ Con la salvedad quizás de que en los textos de Taruffo suelen encontrarse referencias esporádicas a la importancia de que los jueces de los hechos, como agentes principales del sistema de indagación, se conduzcan de modo responsable y comprometido en la búsqueda de la verdad de los hechos controvertidos en juicio. Sin embargo, a pesar de la ubicuidad del tema del juez en sus trabajos, el enfoque en aquellos de sus textos más cargados epistemológicamente se centra sin duda en el sistema probatorio y no tanto en el juez.
} 
En lugar de ocuparse de los estándares de prueba, el principio de inclusión o las cargas probatorias, la ECA reflexiona sobre el tipo de agente (el tipo de jueces) necesario para que la tarea epistémica de resolver acertadamente las cuestiones de hecho debatidas en el juicio se lleve a cabo exitosamente (esto es, llegar a la verdad de los hechos controvertidos que luego se subsumirán bajo las hipótesis de derecho pertinentes, a partir de lo cual finalmente se extraerán y ejecutarán las consecuencias de derecho correspondientes al caso). ${ }^{4}$ Un ejemplo de proyecto filosófico con un enfoque de este tipo se encuentra en los trabajos de Amaya (2008, 2009 y 2012). Asimismo, se identifican algunos rastros de un enfoque con estas características en Malem Seña 2009.

Para entender mejor la diferencia entre ambos modelos imaginemos que el sistema probatorio fuese como una máquina en la cual introducimos ciertos insumos (material probatorio) y de la que esperamos un producto destilado (resultado probatorio o decisión en materia de hechos). La ECS sería una manera de someter dicha máquina y sus componentes a examen para evaluar si están funcionando correctamente (corroborar que el producto obtenido sean resultados probatorios verdaderos). En cambio, la ECA no sería un examen de la máquina, sino más bien un examen del operador de la máquina (el juez de los hechos).

Una vez expuestos estos dos modelos abstractos de epistemología jurídica, ${ }^{5}$ lo que haré ahora será explorar una propuesta epistemológica concreta y examinar la viabilidad de su aplicación al caso jurídico.

${ }^{4}$ Claro que aquí estoy apelando al viejo esquema del silogismo judicial que, si bien es en cierto grado sobresimplificador, sigue siendo una buena herramienta pedagógica para comprender los elementos de la decisión judicial. Entonces, como es bien sabido, la decisión judicial puede representarse como la conclusión de una operación lógica de subsunción de una premisa menor o un cúmulo de premisas menores (premisas sobre los hechos) en una premisa mayor o un cúmulo de premisas mayores (premisas sobre el derecho). El ámbito de la epistemología jurídica, tal y como aquí la concebimos, queda entonces enmarcado por el estudio de los temas relacionados con la correcta "construcción" de la(s) premisa(s) menor(es) del razonamiento judicial. Es la aplicación de la epistemología general a la tarea epistémica o cognitiva de esclarecer cuáles habrán de ser los insumos fácticos en los que habrá de basarse una decisión judicial (al menos las decisiones típicas de los órganos jurisdiccionales de primera instancia). Tales insumos fácticos no deben ser sino la versión verdadera de los hechos que están en la base de la litis, o al menos ése es el desiderátum.

${ }^{5}$ Debe entenderse que hablo de modelos "abstractos" porque no me refiero con estas etiquetas a propuestas epistemológicas en específico, sino a formas o esquemas generales que una epistemología específica puede adoptar. Asimismo, ambos modelos deben verse como los dos polos o extremos de una escala hipotética de opciones teóricas posibles y, como tales, no tienen que corresponder necesariamente a ninguna oferta epistemológica real defendida por algún autor o autora. 
Tal propuesta ha cobrado mucha relevancia en las discusiones filosóficas contemporáneas y se conoce como "epistemología de virtudes". Como se verá, la epistemología de virtudes ( $\mathrm{EV}$, en adelante) es un proyecto epistemológico que, de llevarse al derecho, encuadra dentro del modelo de la epistemología centrada en el agente.

La tarea que emprenderé será, primero, explicar a grandes rasgos en qué consiste la EV y qué rasgos debe presentar en cuanto propuesta de epistemología aplicada al derecho. Luego mostraré en qué falla la EV, es decir, por qué es inviable por sí sola para cumplir la tarea central de la epistemología jurídica (i.e., mostrar cómo es posible el conocimiento de los hechos debatidos en el juicio mediante la admisión, la práctica y la valoración de las pruebas en el marco de un proceso judicial). Al finalizar, mostraré que la EV como modelo de ECA requiere la perspectiva de la ECS para poder ser una epistemología jurídica correcta y que, del mismo modo, la ECS tampoco es suficiente por sí misma y requiere la perspectiva de la ECA para ser una epistemología jurídica completa. A partir de ello concluiré que sólo se puede obtener un modelo satisfactorio de epistemología aplicada al derecho mediante la conjunción de ambas perspectivas.

\section{La EV y sus variedades}

La discusión contemporánea sobre la EV tiene su origen en Sosa 1980. En dicho texto, Ernesto Sosa (quien es hoy tal vez el más influyente exponente de la EV) propone que la epistemología realice un cambio en su análisis tal como el ocurrido en la filosofía moral con el resurgimiento de la ética de virtudes. Tal giro o modificación en el análisis permitiría, según el diagnóstico de Sosa, superar el enfrascamiento en el que había caído la epistemología con el debate entre las posturas coherentistas y fundacionistas de la arquitectura lógica de la justificación epistémica.

Sin entrar en mucho detalle, podemos decir que la modificación en el análisis que propone la ética de virtudes en la filosofía moral es la siguiente: las posturas clásicas del consecuencialismo y el deontologismo tienen por objeto responder la pregunta sobre la acción correcta. Son concepciones de la moral centradas en la acción. La ética de virtudes, en cambio, se enfoca más bien en la pregunta sobre qué tipo de persona debo ser. Dicho de otro modo, las teorías morales tradicionales de corte deontologista o consecuencialista centran su análisis en las acciones y sus criterios de corrección moral. La ética de virtudes, en cambio, pone al agente en el centro del análisis y se 
ocupa más bien de definir cuáles son las cualidades de una buena persona.

Paralelamente, en epistemología el cambio de enfoque en el análisis que abandera la EV en varias de sus versiones (sobre ellas hablaremos más adelante) consiste en poner al agente en el centro del análisis epistemológico. En su forma tradicional contemporánea (confeccionada por medio de los debates en torno al análisis tripartito del conocimiento), la epistemología está centrada en las creencias. Su objeto es identificar qué condiciones debe cumplir una creencia para ostentar el rango de conocimiento (que según el análisis tripartito son, como sabemos, las condiciones de verdad y justificación). En contraste, la EV no se fija tanto en las condiciones de las creencias como en las cualidades intelectuales del agente que le permiten entablar un contacto cognitivo exitoso con el mundo. Aquello que explica la posibilidad del conocimiento según la perspectiva de la EV es el ejercicio de las llamadas "virtudes intelectuales" o "virtudes epistémicas" (de ahí el nombre de esta familia de teorías), que no son sino una serie de excelencias cognitivas, facultades o disposiciones que habilitan a los agentes para conocer.

Dado el giro en el análisis epistemológico que la EV supone, si se intenta llevar al derecho, el resultado sería un tipo de ECA. Ahora bien, para entender mejor esta idea de cambio en el análisis y mejorar nuestra comprensión de la imagen que del conocimiento se tiene en la EV, conviene hacer un breve repaso de las múltiples variedades que ese tipo de teoría puede adoptar. ${ }^{6}$ Ésta es, además, una tarea de particular importancia para los efectos de este trabajo, pues en él no tengo en mente cualquier versión de EV, sino una versión específica cuya viabilidad como epistemología del derecho quiero poner en tela de juicio.

Así pues, debemos comenzar por reconocer que en la EV hay una primera gran dicotomía a partir de la manera en que los diversos filósofos entienden la noción misma de virtud epistémica. Por un lado, tenemos la llamada concepción fiabilista de las virtudes (virtue reliabilism $),{ }^{7}$ según la cual las virtudes se deben entender como

\footnotetext{
${ }^{6}$ La clasificación que elaboraré aquí no es novedosa ni única. Tampoco debe tomarse como una división estricta asumida o trabajada por todos los autores en la literatura especializada, sino únicamente como una taxonomía basada en mi comprensión del estado actual del arte. Para ello seguiré de cerca, aunque no estrictamente en sus términos, la clasificación que se propone en Baehr 2011, pp. 9-12.

${ }^{7}$ Entre los filósofos más prominentes de línea fiabilista están el propio Ernesto Sosa (1991, 1992 y 2007), Alvin Goldman (1992 y 2001) y John Greco (1992, 2002 y 2010).
} 
facultades cognitivas fiables (esto es, conductoras de verdad en la mayoría de los casos). Siguiendo esta línea, algunos ejemplos de virtudes intelectuales serían la memoria, la vista, el oído, la capacidad de razonar deductivamente, por mencionar algunas. Entonces, según el fiabilismo, el conocimiento se explica como el resultado de la intervención de estas competencias o habilidades epistémicas, las cuales permiten, por su fiabilidad, que los agentes lleguen a tener creencias verdaderas y justificadas.

Ahora bien, no todos los filósofos conciben las virtudes epistémicas de esa manera. En respuesta a la propuesta de Sosa en "The Raft and the Pyramid" (1980), Lorraine Code publica su célebre libro Epistemic Responsibility (1987). Éste representa el primer trabajo en el que se expone una forma de $\mathrm{EV}$ conocida como responsabilismo, ${ }^{8}$ de acuerdo con la cual las virtudes epistémicas más que entenderse como facultades cognitivas, se entienden como rasgos del carácter de las personas con implicancias en la manera en que afrontan y resuelven las tareas epistémicas que emprenden. Entonces, de acuerdo con el responsabilismo, la noción de virtudes epistémicas se refiere a los equivalentes intelectuales de las virtudes morales tradicionales como la humildad, la tenacidad y la sobriedad. Poseer y ejercer esas cualidades, según el responsabilismo, nos hace mejores agentes epistémicos, pues potencia nuestro desempeño en tareas epistémicas más complejas e interesantes como la reflexión filosófica, la indagación científica o, por supuesto, la prueba de los hechos en un proceso judicial.

Lo que subyace en el fondo de la dicotomía fiabilismo/responsabilismo es una diferencia en las intuiciones sobre lo que es necesario para el conocimiento. Así, los fiabilistas piensan que lo necesario para que haya conocimiento es que éste sea producto del ejercicio de ciertas facultades que de modo estable y regular tienden a llevar con mucha mayor frecuencia a verdades que a falsedades. En cambio, a los responsabilistas les interesan más las cuestiones relativas a la responsabilidad y los deberes epistémicos que los agentes tienen como productores de conocimiento. Los responsabilistas suelen estar más preocupados por asuntos como nuestros deberes doxásticos qua agentes epistémicos. Esto hace del responsabilismo una línea de ideas interesante para el derecho. Parece importante señalar que una tarea epistémica con tanta relevancia social y política como la determina-

${ }^{8}$ Además de Lorraine Code (1987), otros autores destacados en la corriente responsabilista son James Montmarquet (1993) y Linda Zagzebski (1996), la exponente más relevante de esta línea de pensamiento en el estado actual del debate. 
ción que un juez o jueza toma respecto de cuál versión de los hechos declarará como probada en un proceso supone que —además de que preocupa que esa versión sea verdadera - nos preocupa que se llegue a ella de buen modo (con meticulosidad, sobriedad, compromiso, etc.). Sobre eso hablaremos párrafos más adelante.

Una segunda dicotomía en la cual se pueden clasificar las variedades de EV (sean fiabilistas o responsabilistas) es entre EV rutinaria y EV autónoma. La diferencia aquí radica en el tipo de problemas epistemológicos que se pueden abordar apelando a la idea de virtudes epistémicas. Por un lado, se puede utilizar el esquema de virtudes para abordar los problemas tradicionales de la epistemología como el problema del conocimiento, la suerte epistémica, la justificación y el escepticismo. ${ }^{9}$ Esto es lo que hace la EV rutinaria. Por otro lado, el esquema de las virtudes intelectuales se puede utilizar para enfocarse en problemas y conceptos no centrales ${ }^{10}$ y usualmente olvidados por la epistemología tradicional, como el entendimiento y la sabiduría. Proyectos con estos rasgos integrarían la llamada EV autónoma.

Por último, una dupla más de variedades o formas que puede adoptar la EV son la robusta (EVR, en adelante) y la austera (EVA, en adelante). Ambas pueden presentarse como subespecies de la EV rutinaria y de la EV autónoma; como subespecies de la EV rutinaria, responden a problemas epistemológicos tradicionales, con la diferencia de que la EVR defiende el papel central de las virtudes epistémicas en la solución de dichas cuestiones, mientras que la EVA asume que las virtudes tienen un papel secundario o auxiliar en el tratamiento de los problemas epistemológicos centrales que se señalaron antes. Por su parte, como subespecie de la EV autónoma, una teoría se puede clasificar como EVR si su propuesta consiste en sustituir los problemas centrales o tradicionales de la epistemología contemporánea para poner al centro nuevos problemas y discusiones antes olvidados o tachados de irrelevantes. Equivalentemente, una EV autónoma puede adoptar la forma de EVA si su propuesta es más modesta y con ella no se pretende sustituir o reformar las discusiones tradicionales, sino sólo complementarlas o ampliar el espectro

\footnotetext{
${ }^{9}$ Ésas son las tareas que han emprendido filósofos como Sosa, Zagzebski y Greco mediante la epistemología de virtudes.

${ }^{10}$ El trabajo de Lorraine Code, por ejemplo, es una EV abiertamente pensada sin la intención de abordar los temas habituales de la epistemología tradicional. Del mismo modo Roberts y Wood (2007) proponen un esquema de EV cuya finalidad no es tratar las cuestiones centrales de la teoría del conocimiento.
} 
de las cuestiones que aborda la epistemología misma.

El caso de la EVR es, en concreto, el tipo de EV que quiero evaluar en este trabajo. Las dos versiones más influyentes de EVR son las de Ernesto Sosa (2007) y Linda Zagzebski (1996); fiabilista la primera y responsabilista la segunda. En la siguiente subsección esbozaré un esquema de la EVR con elementos responsabilistas y mostraré cómo se aplica ese modelo al caso jurídico.

El siguiente cuadro resume las variedades de EV que acabo de exponer en esta sección.

\begin{tabular}{|c|c|c|}
\hline \multicolumn{3}{|c|}{ EPISTEMOLOGÍA DE VIRTUDES } \\
\hline $\begin{array}{c}\text { Según su concepción } \\
\text { de las virtudes }\end{array}$ & $\begin{array}{c}\text { Según su posición } \\
\text { respecto de los temas } \\
\text { centrales }\end{array}$ & $\begin{array}{c}\text { Según sus pretensiones } \\
\text { como teoría } \\
\text { del conocimiento }\end{array}$ \\
\hline Fiabilista & Rutinaria & Robusta \\
\hline Responsabilista & Autónoma & Austera \\
\hline
\end{tabular}

2.1. ¿Cuál es la EV para el ámbito probatorio?

Vistas las diversas construcciones que el esquema teórico de la EV puede adoptar, procederé a presentar la estructura general de una versión ambiciosa de EV que pueda plantearse como candidata a ser la epistemología estándar del derecho. Una vez hecho eso, mostraré en las secciones subsiguientes los problemas que enfrenta un proyecto así y por qué no se sostiene como epistemología general o estándar en el derecho.

Pues bien, para determinar qué rasgos debe reunir una EV adecuada para nuestros fines, es necesario comenzar por identificar qué rasgos presenta la prueba de los hechos como tipo de indagación. En este tenor, lo primero que hay que notar es que en un proceso judicial no todo cuenta como prueba. Hay reglas y criterios que definen qué tipo de materiales son admisibles jurídicamente como pruebas y cuáles no. Lo habitual es que en este terreno se maneje un principio general de admisión, es decir, un principio de apertura a todo tipo de materiales potencialmente relevantes. Sin embargo, a este principio general de admisión también se le imponen con frecuencia varias excepciones como, por ejemplo, la constitucionalidad de determinada pieza de material probatorio (i.e., que no se haya obtenido vulnerando algún derecho fundamental) o el respeto al principio de economía 
procesal en virtud del cual las pruebas redundantes $\mathrm{u}$ ociosas, aunque potencialmente relevantes, deben quedar fuera. ${ }^{11}$

Aunado a lo anterior, también debemos tomar nota de que, para los efectos de su decisión, el o los agentes epistémicos encargados de resolver el caso (que bien pueden ser un juez, un grupo de jueces o un jurado) están obligados a tomar en consideración única y exclusivamente los materiales probatorios que han sido integrados al expediente judicial en tiempo y forma. Sólo lo que consta en autos cuenta como prueba para los efectos de la decisión judicial en materia de hechos. Este constreñimiento es una peculiaridad de la prueba de los hechos en el derecho que tiene una importancia cardinal en razón de las modificaciones en el nivel epistémico que su operatividad supone.

Por último, debemos estar conscientes del tipo y la calidad de información que el órgano jurisdiccional posee al momento de decidir. Por un lado, una gran cantidad de la información puesta a disposición del juez es información de "segunda mano", valga la expresión. Las pruebas, en buena medida, son información que el juez recibe de otros agentes. El juez, por definición, no puede ser alguien que haya conocido directamente los hechos que se someten a su consideración (de lo contrario no sería juez, sino testigo). Esto significa que la cognición del juez es, en sentido lato, testimonial. El juez se enfrenta ni más ni menos que a la difícil tarea de conocer un hecho o un cúmulo de hechos echando mano de lo que otros le dicen al respecto. Por otro lado, las partes no son agentes neutrales en una litis. Las partes tienen intereses en juego y no es de sorprender que la información que transmiten al juez pase por el filtro de sus intereses e intenciones. Esto quiere decir que el conjunto de insumos probatorios con los que cuenta el juez al momento de decidir cuáles fueron los hechos no es necesariamente un conjunto de pruebas plenamente fiable.

A la dificultad de obtener un cúmulo probatorio fiable sólo restaría agregar la dificultad de que sea completo. Por un lado, el sistema probatorio suele autorizar que las partes planteen ante el tribunal "hechos no controvertidos", es decir, hechos que ninguna de las partes disputa y de los cuales, en consecuencia, no se suele presentar prueba o, si se presenta, se desecha en pro de la economía procesal. Además, el proceso judicial no puede proseguir indefinidamente; es algo que

${ }^{11}$ Quizás sobra la aclaración, pero es importante recordar el sentido técnico de la noción de relevancia en el ámbito probatorio: determinada prueba $K$ es relevante para los efectos de probar la proposición $P$ si y sólo si la presentación de $K$ incrementa las probabilidades de que $P$ sea verdadera. 
en un momento debe concluir. El juez tiene el deber de resolver el asunto en un plazo razonable o en el que fije la ley, de modo que la indagación sobre los hechos, como parte del proceso general, está sometida a un plazo y debe concluir en algún punto. Por operación del constreñimiento epistémico, esa conclusión se debe realizar en el tiempo establecido y sin contar más que con los materiales que constan en autos.

Todo lo anterior nos arroja sólo un panorama general de la complejidad y dificultad de arribar a la verdad en el contexto epistémico que nos ocupa. ¿Implica esto abandonar la esperanza de que la verdad sea posible en el ámbito probatorio judicial? No. Aun a pesar de las dificultades planteadas (y otras que puedan sugerirse), la verdad en la decisión probatoria es en principio posible. Salvo que se adopte alguna forma de escepticismo más radical, lo que he esbozado aquí es, creo, sólo una tarea difícil y no imposible.

Ante tal panorama, lo que prosigue es definir los elementos que compondrían una aproximación dentro de las líneas de la EV que pueda funcionar para explicar la posibilidad de conocer en el contexto en cuestión. Para comenzar, cuando se asume como tarea fundamental explicar la posibilidad del conocimiento, se dejan de lado las EV autónomas pues se está adoptando una cuestión epistemológica central o tradicional como problema por elucidar. Eso significa que la epistemología que presentaré aquí será una EV rutinaria.

Aclarado eso, conviene ahora preguntarnos si es viable una EV fiabilista. Recordemos que, de acuerdo con esa corriente, aquello que explica el conocimiento es el ejercicio de las virtudes entendidas como facultades cognitivas. Y a golpe de vista todo indica que, en efecto, las facultades cognitivas como la memoria, la vista y la capacidad de razonar deductivamente desempeñan algún papel en la tarea epistémica a la que se enfrentan los jueces de los hechos. Sin embargo, el fiabilismo de virtudes es un esquema pensado para casos diferentes de conocimiento. Es un esquema teórico que sirve para explicar nuestro conocimiento de proposiciones como "Hay un monitor de computadora delante de mí" o "Esta mesa es blanca". Sin embargo, la tarea encomendada a los jueces de los hechos es más compleja y queda la sensación de que el aparato conceptual del fiabilismo se queda corto para dar cuenta de ella.

Dadas las complejidades y dificultades mencionadas, resulta evidente que el ámbito probatorio es un contexto epistémico cuyo conocimiento será posible sólo si operan más cosas y no sólo las meras habilidades cognitivas del agente. La prueba de los hechos en el derecho exige un compromiso con la verdad, el esfuerzo y la dedicación, 
entre otras. Esta tarea exige que el agente sopese con cuidado los argumentos y la información que se le presentan (meticulosidad o cuidado intelectual), que no claudique en sus esfuerzos por esclarecer los hechos cuando tiene la sana y correcta convicción de que hay más por descubrir que lo que está a la vista (tenacidad intelectual), y que se abstenga de realizar saltos inferenciales apresurados o de caer en la credulidad (sobriedad intelectual). De igual modo, los jueces deben estar abiertos a la crítica y a las sugerencias de otros, a mantener un espíritu activamente autocrítico y a no sobreestimar sus capacidades intelectuales (humildad intelectual).

En vista de todo lo anterior, el proyecto de EV que a primera vista encuentro viable para el derecho es de índole responsabilista, en razón de que los rasgos del carácter que destaca el responsabilismo definen mejor el perfil de un buen juez y ofrecen un aparato conceptual más fecundo para explicar cómo pueden los jueces, en principio, llegar al conocimiento de los hechos. Sin embargo, si somos un poco más exigentes, el proyecto que tengo en mente puede ser más bien mixto, pues no tiene por qué negar la relevancia que naturalmente tienen las habilidades cognitivas (como la memoria o la vista) para la tarea en cuestión. Sólo que ocurre que el ámbito probatorio, una indagación propiamente dicha, ${ }^{12}$ requiere más que eso para producir conocimiento.

Una vez que se ha definido que el proyecto que estoy desarrollando aquí es una EV responsabilista y rutinaria, lo que queda es revisar qué ocurre si se opta por formularla como una versión de EVR. Esto significaría que, desde la perspectiva de ese modelo epistemológico (que, dicho sea de paso, sería un modelo de ECA), el conocimiento que los jueces llegan a tener de los hechos que suscitaron una controversia de intereses jurídicos se debe a que ejercen virtudes intelectuales. Cosa que, así dicha, no es menor, pues supone que si tenemos jueces virtuosos, entonces estamos en posibilidades de obtener mejores decisiones. Lo que nos debe preocupar entonces es tener jueces con esas competencias epistémicas, ya sea porque los busquemos así y luego los nombremos o, mejor aún, porque los formemos así.

Ahora bien, por muy provocador que esto suene, veo muy improbable que el conocimiento de los hechos en un proceso judicial pueda

\footnotetext{
${ }^{12}$ Como acertadamente expresa Baehr: "la indagación supone una dimensión vigorosamente activa. Es algo que demanda observar, imaginar, leer, interpretar, reflexionar, analizar, evaluar, conjeturar y articular" (2011, p. 1; la traducción es mía, las cursivas del autor).
} 
explicarse apelando únicamente a elementos de una teoría de virtudes epistémicas. Lo que haré a continuación es mostrar una objeción importante a la EVR en la epistemología general para luego mostrar cómo se traduce en el terreno jurídico. ${ }^{13}$

\section{Casos Gettier y el argumento contra la suerte epistémica}

El análisis tripartito del conocimiento se vio severamente socavado por Edmund Gettier (1963) y sus famosos contraejemplos. El análisis tripartito, tal y como se entendía en la literatura previa a Gettier, se veía así:

$$
\begin{aligned}
S \text { sabe que } P \leftrightarrow & \text { (i) } S \text { cree que } P \\
& \text { (ii) Es verdad que } P \\
& \text { (iii) } S \text { está justificado para creer que } P
\end{aligned}
$$

De acuerdo con tal análisis, las condiciones necesarias y suficientes para tener conocimiento proposicional son la creencia, la verdad de dicha creencia y que ésta se encuentre justificada epistémicamente.

Una intuición importante en la epistemología es que el conocimiento es incompatible con la suerte. Supongamos que yo decido lanzar una moneda al aire. Si cae cara creeré "Hoy es miércoles". Si cae cruz creeré "Hoy es jueves". Lanzo la moneda, cae cara y creo que es miércoles. Ahora bien, aun si resultase que hoy en efecto es miércoles, la verdad de mi creencia no se debería más que al azar.

${ }^{13}$ Como ya lo he señalado, la epistemología de virtudes robusta es una propuesta teórica que se ha discutido con relativa efervescencia en la literatura filosófica de nuestros días. El objetivo de discutirla aquí es descubrir si se trata de un proyecto viable en cuanto a su aplicación a la epistemología del derecho o si se debe descartar. En el terreno jurídico no existe una propuesta de aplicación de este corte que se pueda definir plenamente. Tal vez esto se deba en buena medida a que la literatura sobre epistemología de virtudes aplicada al derecho es escasa. Pero por la vigencia que la EVR tiene en el debate epistemológico actual, creo que merece la pena dedicar un espacio a reflexionar sobre su viabilidad o inviabilidad en la epistemología jurídica, sea el caso o no que se trate de una postura que en efecto defienda algún autor o autora en concreto. Es una opción teórica concebible, en principio, y mi objetivo aquí es examinarla. Para una mayor discusión filosófica sobre la EVR, véanse Pritchard 2010a, pp. 25-28 y 2010b; Vaesen 2011; Kallestrup y Pritchard 2012; Kelp 2013; Broncano-Berrocal 2016, y Carter 2016. Por su parte, Baehr (2011) llama a esta opción teórica "epistemología de virtudes fuerte" (y se la atribuye a Zagzebski 1996). Amaya (2008, p. 314), al abordar la justificación de la decisión probatoria en el derecho, también habla de una teoría de virtudes fuerte. 
Eso significa que creer algo, aunque sea verdadero, no es suficiente para que esa creencia sea conocimiento. Así, entonces, el análisis tripartito parecería evadir el problema de la suerte epistémica al incluir la justificación. Sin embargo, el reto que los casos Gettier plantean es que se pueden cumplir las tres condiciones del análisis tripartito y aun así no tener conocimiento. En otras palabras, un caso Gettier es aquel en el que tenemos una creencia verdadera y justificada, pero aun así ello nos resistiríamos a atribuir conocimiento a quien ostenta dicha creencia. Veamos un ejemplo:

El miércoles 23 de septiembre de 2015 se juega en Barcelona la final del Campeonato Mundial de Clubes de Protobol 2015 de la Asociación Internacional de Federaciones de Protobol (PIFA, por sus siglas en inglés). Los contendientes son ni más ni menos que los escurridizos Pulpos de Galicia y las asombrosas Cacatúas Púrpuras de Veracruz. Yo, acérrimo seguidor de estas últimas, sintonizo el canal de televisión en el que las he seguido a lo largo del torneo y donde sé que transmitirán la gran final. Me siento en el sofá, apago el teléfono móvil y me dispongo a ver el partido. El partido finaliza y las Cacatúas pierden 2-1 contra los Pulpos, quienes se alzan con la victoria.

En razón de lo anterior, me formo la creencia de que los Pulpos de Galicia son los campeones del Mundial de Clubes de Protobol 2015 de la PIFA. Y es verdad: las Cacatúas perdieron 2-1 contra los Pulpos y los Pulpos son los campeones del 2015. Además, mi creencia está justificada, pues lo vi por televisión y ésa es una fuente fiable para la formación de creencias en este tipo de asuntos. Sin embargo, el canal tuvo un problema súbito con la transmisión del partido, ante lo cual, sin saberlo yo, se vieron obligados a transmitir la final del año pasado que afortunadamente también disputaron en Barcelona los Pulpos y las Cacatúas. De hecho, el año anterior los Pulpos también ganaron 2-1. Entonces, ocurre que mi creencia cumple las condiciones que el análisis demanda (creencia, verdad, justificación), pero yo no sé que los Pulpos de Galicia son los campeones de protobol 2015, pues la verdad de mi creencia no se debe más que a la buena fortuna.

Lo que ocurre en un caso Gettier es que, por culpa de la mala fortuna, nuestro compás epistémico se ve estropeado y la nave de nuestras creencias se desvía de su rumbo. Pero, por intervención de la buena fortuna, los vientos llevan nuestra nave al puerto esperado (la verdad) aunque el compás haya funcionado mal. Lamentablemente, aun 
cuando nuestra creencia sea verdadera y esté justificada, no se nos puede atribuir conocimiento.

La respuesta de la EVR al reto de Gettier es indicar que el problema radica en que la verdad de la creencia en juego no está ocasionada por el ejercicio de las virtudes intelectuales ni es producto de ellas. Según la EVR, el conocimiento se debe entender como el resultado de la utilización exitosa de nuestras competencias intelectuales (sean facultades o sean rasgos del carácter).

En términos de la EVR que propone Ernesto Sosa (2007), por ejemplo, el conocimiento es una creencia óptima. Ese estatus se obtiene a partir de la conjunción de dos elementos: destreza y precisión. Destreza es lo que supone el ejercicio de nuestras competencias epistémicas, pues son las cualidades que nos habilitan para conocer. Precisión es el requisito de éxito epistémico, esto es, la capacidad de dar con la verdad. En un caso Gettier, lo que ocurre es que la creencia no es óptima dado que su precisión no es el resultado de nuestra destreza. La verdad de la creencia no está ocasionada por el ejercicio de la virtud. Esto significa que, según la EVR, el conocimiento exige una conexión causal apropiada entre una creencia y su verdad y que dicha conexión se establezca mediante la virtud epistémica. En un caso Gettier, dicha conexión no se satisface y, en consecuencia, no hay conocimiento.

Esta respuesta que da la EVR a Gettier, aunque parece plausible a primera vista, ha sido atacada por filósofos como Baehr (2011, pp. 3639) y Kallestrup y Pritchard (2012). El problema que se plantea es básicamente el siguiente: en efecto, si bien la verdad de la creencia en cuestión en un caso Gettier no está causada por la virtud intelectual ni es producto de ella, el ejercicio de esta última es lo que explica que la creencia esté justificada en primer lugar. Es la intervención de nuestras competencias epistémicas lo que produce y justifica la creencia. Simplemente ocurre que el ejercicio de las virtudes no es lo que produce la verdad ni la creencia y, en consecuencia, aun cuando se cumplan las condiciones planteadas por la EVR para el conocimiento, éste no existe en ese caso.

Es fácil gettierizar la concepción del conocimiento que tiene la EVR. Veamos:

Imaginemos el caso de una especialista en el diagnóstico de enfermedades raras. Se ha ganado el respeto de sus colegas del hospital por la pulcritud de su trabajo, e incluso el reconocimiento como autoridad internacional en su especialidad. Es una investigadora tenaz, docta, autocrítica, de mente abierta, sobria, 
lúcida, razonable, etc. Es, sin más, desde el punto de vista epistémico, una persona virtuosa como pocas. Supongamos que un día toma el caso de una paciente y, como es habitual en ella, inicia una indagación cuidadosa en la que sigue todos los protocolos clínicos estándar (varios de ellos de su autoría). Entre las múltiples tareas que tiene entre manos, decide realizar una especie particular de exámenes de laboratorio. Tras tomar las muestras correspondientes, las envía a un afamado laboratorio de la ciudad y solicita que se apliquen las pruebas. Tragicómicamente, quien recibe y etiqueta las muestras ese día es un pasante en su primer día de trabajo en el laboratorio. Dada su inexperiencia, este chico confunde las muestras enviadas por la especialista con otras que se acaban de recibir y cambia las etiquetas. Tiempo después, una vez realizadas las pruebas de laboratorio, los resultados llegan al hospital y la doctora llega a la conclusión de que, por toda la evidencia disponible (incluidas las pruebas de laboratorio recién recibidas), la paciente tiene lupus. Recalquemos: dicho diagnóstico toma en consideración los resultados de laboratorio correspondientes a unas muestras tomadas de otra persona. Sin embargo, por fortuna, la paciente ¡de hecho tiene lupus! (no tanta fortuna para ella, sin duda, ni tampoco para el "autor" de las otras muestras, quien esperemos que no tenga lupus también). Esto significa que el diagnóstico ha sido exitoso, en cuanto a que ha sido verdadero. Además, es el resultado de una indagación genuinamente virtuosa por parte de la especialista en cuestión.

Lo que este segundo ejemplo señala es lo ya dicho. Aunque las virtudes estén presentes y el resultado de la indagación sea en buena medida producto de su intervención, lo cierto es que la verdad del resultado no está causada por las virtudes de quien indaga sino por la mera fortuna epistémica. En efecto, como sostiene la EVR, puesto que la verdad de la creencia no se debe a la virtud, entonces no estamos ante un caso de conocimiento. Sin embargo, estamos ante un caso de creencia verdadera afortunadamente, que además está justificada por la intervención de la virtud. Después de todo, el proceder epistémico de la agente en el ejemplo fue sencillamente irreprochable. Esto lleva a suponer que, además del ejercicio de las virtudes intelectuales, hace falta algo para que haya conocimiento. La EVR no supera el problema de Gettier.

El meollo del problema yace en la accidentalidad de la verdad de la creencia. Ocurre que algo nos hace falta, algo que permita que la 
verdad de nuestras creencias se produzca por la operación de las virtudes. Es necesario que la verdad sea no accidental. Necesitamos que las condiciones en las cuales se gesta la creencia sean tales que se permita que las virtudes operen apropiadamente. Sin las condiciones apropiadas, las virtudes no son garantía de verdad en el resultado de una indagación.

\section{Gettierizando la prueba de los hechos}

Las implicaciones del problema que hemos planteado al intentar aplicar la EVR al derecho son evidentes. Como he apuntado, el ejercicio de las virtudes por parte de un juez no garantiza por sí solo que el resultado probatorio sea verdadero si las condiciones no son las apropiadas. Incluso es posible que en tal contexto epistémico ocurran situaciones de tipo Gettier. ${ }^{14}$

Hay que recordar lo dicho párrafos antes sobre las peculiaridades que caracterizan al contexto probatorio como contexto de indagación. Las partes pueden "maquillar los hechos", ofrecer interpretaciones de las pruebas que convengan a sus intereses y pretensiones, ocultar información o tergiversarla, pueden incluso fabricar pruebas, aleccionar a los testigos, contratar expertos que emitan peritajes que les favorezcan. Los abogados a veces pueden ser verdaderos artesanos del engaño y engañar a los jueces sin que éstos tengan manera de sospechar que algo anda mal.

Fácilmente puede ocurrir, y con toda seguridad ha ocurrido más de una vez, que algún fiscal manipule las pruebas de modo tal que la culpabilidad de un acusado aparente ser indudable, que, además, ese acusado de hecho sea culpable (sólo que no en los términos de la versión de los hechos que el juez declara probada), que el juez haya desplegado una conducta epistémica irreprochable en su seguimiento del caso y que, además, crea que el acusado es culpable, lo cual, para la buena suerte epistémica del juez, es verdad. Los casos Gettier tal vez son una realidad cotidiana en los juzgados.

Ahora bien, se puede adoptar una política "resultadista" y decir “¿Qué más da si es accidental la verdad del resultado? Lo importante es que sea verdad y punto." Ése es un tema que por razones de economía expositiva no abordaré aquí. Pero sí señalaré que el problema de no hacer nada para erradicar la suerte epistémica es que, como vimos, su presencia es indicativa de que algo no anda bien en las

${ }^{14} \mathrm{La}$ posibilidad de que se generen resultados probatorios verdaderos por mera fortuna se contempla plenamente en la literatura especializada. Véanse Pardo 2010, p. 42, y 2005, pp. 322-323; Duff, Farmer et al. 2004, pp. 1-28. 
condiciones de indagación. Aunque la fortuna a veces nos sonría, con toda seguridad llegará el día en que nos apuñale por la espalda. Entonces, si bien tener un resultado verdadero es ciertamente mejor que tener uno falso, es de importancia primaria que nos encarguemos de pulir las condiciones de indagación de modo que esa verdad se alcance y no por accidente.

La persistencia del problema de Gettier y la incapacidad de la EVR para superarlo anuncian su fracaso como proyecto epistemológico rutinario. Asimismo, la persistencia del problema de Gettier en el ámbito jurídico sirve para diagnosticar su inaplicabilidad plena a dicho contexto. Todo esto da muestra de la necesidad de realizar un cambio en la posición. Las opciones son o bien abandonar la EV por completo o replegarnos a una versión más plausible de ella (EVA).

\section{La inaplicabilidad de la EVR como teoría del conocimiento en el ámbito probatorio. Moralejas para un nuevo modelo de epistemología aplicada al derecho}

La EVR falla entonces como epistemología general, y es de esperar que falle como epistemología en la provincia del derecho en particular. Como vimos, lo que ocurre es que el ejercicio de las virtudes epistémicas no basta por sí solo para hacer posible el conocimiento. En consecuencia, la posición se ha de enmendar o abandonar.

En el caso del derecho, lo que ocurre con las virtudes es que, si bien conservan un papel importante en cuanto garantes de la justificación epistémica, no son garantes de la verdad del resultado. $\mathrm{Y}$ no lo son porque las condiciones del contexto no permiten que lo sean. Pero, ¿qué son esas "condiciones"? Pues no son otra cosa que el sistema probatorio mismo. Las reglas del sistema son las que definen qué cuenta como pruebas, quién las aporta y cómo se razona a partir de ellas. Si las reglas permiten engañar al juez o limitar significativamente sus opciones, entonces, por virtuoso que sea, el resultado de su indagación no tendrá garantía de verdad.

El sistema probatorio es, entonces, el cúmulo de condiciones necesarias para la obtención de la verdad sobre los hechos. Si está configurado correctamente, el sistema operará como un conjunto de condiciones de indagación habilitantes para la obtención de conocimiento. El sistema es el espacio en el que se mueve la virtud y para que se mueva con soltura es necesario que el espacio esté acondicionado correctamente. Esto quiere decir que lo relevante desde el punto de vista epistemológico no es la virtud, sino que las condiciones de indagación sean habilitantes epistémicamente. 
Entonces, el fracaso del proyecto de la EVR como epistemología del derecho radica en su incapacidad para mostrar cómo es posible el conocimiento en el contexto probatorio procesal apelando únicamente a los elementos de una teoría de virtudes. Es necesario apelar a la noción de condiciones de indagación; es necesario dirigir la atención hacia el sistema en cuanto cúmulo de condiciones epistémicas que promueven u obstaculizan el descubrimiento de la verdad.

¿Significa este fracaso que debemos dar vuelta a la página y olvidarnos de la EV? No necesariamente. Lo que debemos extraer de este fracaso es sólo que la EVR no es viable y que debemos tornar la mirada hacia una EVA. Si bien la lección es que las virtudes no son suficientes por sí mismas para llegar a la verdad de los hechos controvertidos en un juicio, éstas aún pueden conservar un papel importante. Si operan bajo las condiciones correctas, alcanzaremos el ideal de un resultado probatorio verdadero y gestado de manera irreprochable.

Este fracaso de la EVR apunta, además, a que un modelo de ECA es inapropiado, pues hace falta la mirada del sistema, en la que se centra el modelo de la ECS; sin embargo, la ECS también se queda corta. No podemos abandonar simplemente la perspectiva del agente. A fin de cuentas, como se suele afirmar, un sistema de justicia será tan bueno como las mujeres y los hombres que lo aplican.

La inviabilidad de la EVR, insisto, no implica abandonar por completo la EV, sino una retirada estratégica hacia la EVA. Esto significa que subsiste el deber de seguir reflexionando y trabajando sobre nuestros sistemas probatorios de modo que los afinemos. Una vez expuesto el panorama tal como lo he hecho aquí, es notorio que hay riesgos y problemas que debemos eliminar. Una buena parte ellos se puede combatir si integramos al escenario la figura de un juez virtuoso. La EV permite revivir con nuevos bríos el debate acerca de qué tipo de persona es la que queremos que ocupe el cargo de juez. La EVA, como se ha indicado, no es un tipo de teoría del conocimiento que dé centralidad a la noción de virtud intelectual, sino que le otorga un espacio secundario, pues las preocupaciones en torno a la construcción correcta del sistema probatorio siguen siendo el elemento más importante en términos epistemológicos. Sin embargo, haríamos mal si continuáramos obviando u olvidando el asunto de los jueces y sus competencias intelectuales, como suele ocurrir en los modelos de epistemología centrada en el sistema.

Un proyecto del corte de la EVA, si ha de convertirse en una epistemología jurídica completa y satisfactoria, habrá de brindar 
una perspectiva integral de los problemas epistemológicos que rodean la prueba de los hechos en un juicio. Éstos abarcan desde la construcción del sistema hasta las cualidades y características que deben poseer los jueces como operadores del sistema. Por sus características, la EVA no es ni un modelo de ECA ni de ECS. En realidad se encuentra en un punto intermedio entre ambos polos de la escala teórica hipotética. Si se emprende la tarea de construir un modelo de epistemología con esos rasgos, entonces se combinarán las fortalezas de la perspectiva de los mejores ejemplos de la ECS con la novedosa revitalización del tema de los jueces (habitualmente relegado no sólo en la epistemología del derecho, sino en el derecho procesal). Una versión exitosa de la EVA nos debe dar indicios de los rubros en que debemos trabajar para pulir nuestros procesos de formación de jueces, así como pistas acerca de qué cosas son las que funcionan bien y cuáles mal en nuestro sistema probatorio. Creo que ése es un rumbo adecuado para la reflexión sobre los problemas epistemológicos que se presentan en el seno de la prueba de los hechos durante un proceso judicial. ${ }^{15}$

\section{BIBLIOGRAFÍA}

Amaya, Amalia, 2012, "The Role of Virtue in Legal Justification", en Amalia Amaya y Hock Lai Ho (comps.), Law, Virtue and Justice, Hart, Oxford, pp. 51-66.

—_, 2009, "Inference to the Best Legal Explanation", en Hendrik Kaptein, Henry Prakken y Bart Verheij (comps.), Legal Evidence and Proof: Statistics, Stories, Logic, Ashgate, Aldershot, pp. 135-159.

Amaya, Amalia, 2008, "Justification, Coherence, and Epistemic Responsibility in Legal Fact-Finding", Episteme: Journal of Social Epistemology, vol. 5, no. 3, pp. 306-319.

Baehr, Jason, 2011, The Inquiring Mind. On Intellectual Virtues and Virtue Epistemology, Oxford University Press, Nueva York.

Broncano-Berrocal, Fernando, 2016, "A Robust Enough Virtue Epistemology", Synthese, pp. 1-28, disponible en <https://link.springer.com/ article/10.1007/s11229-016-1043-y >.

${ }^{15}$ Agradezco a Sebastián Figueroa, Jonatan Valenzuela, Carolina Bruna, Verónica Rodríguez-Blanco y Matias Parmigiani por discutir y comentar versiones previas de este artículo. Agradezco también a los miembros del Departamento de Derecho Procesal de la Universidad de Chile por sus múltiples observaciones y aportaciones. Agradezco asimismo a Diego Papayannis y Lorena Ramírez por impulsar el desarrollo de este trabajo. Quedo en deuda con los revisores anónimos de este texto por sus puntuales y meditadas observaciones. 
Carter, J. Adam, 2016, "Robust Virtue Epistemology as Anti-Luck Epistemology: A New Solution", Pacific Philosophical Quarterly, vol. 97, no. 1, pp. 140-155.

Code, Lorraine, 1987, Epistemic Responsibility, Brown University Press, Hanover.

Duff, Antony, Lindsay Farmer et al. (comps.), 2004, The Trial on Trial, vol. 1, Truth and Due Process, Hart, Oxford.

Ferrer Beltrán, Jordi, 2005, Prueba y verdad en el derecho, 2a. ed., Marcial Pons, Madrid.

Gettier, Edmund, 1963, "Is Justified True Belief Knowledge?", Analysis, vol. 23, no. 6, pp. 121-123.

Kallestrup, Jesper y Duncan Pritchard, 2012, "Robust Virtue Epistemology and Epistemic Anti-Individualism", Pacific Philosophical Quarterly, vol. 93, no. 1, pp. 84-103.

Kelp, Christoph, 2013, "Extended Cognition and Robust Virtue Epistemology", Erkenntnis, vol. 72, no. 2, pp. 245-252.

Laudan, Larry, 2016, “¿Por qué las reglas procesales asimétricas hacen imposible calcular un estándar de prueba racionalmente justificado?", en Jordi Ferrer Beltrán y Carmen Vázquez (comps.), Debatiendo con Taruffo, Marcial Pons, Madrid, pp. 341-352.

- 2008, Truth, Error, and Criminal Law: An Essay in Legal Epistemology, Cambridge University Press, Cambridge.

Malem Seña, Jorge Francisco, 2009, “La formación de los jueces: los saberes del juez", en Jorge Francisco Malem Seña, Francisco Javier Ezquiaga Ganuzas y Perfecto Andrés Ibáñez (comps.), El error judicial. La formación de los jueces, Fundación Coloquio Jurídico Europeo, Madrid, pp. 95-126.

Montmarquet, James A., 1993, Epistemic Virtue and Doxastic Responsibility, Rowman and Littlefield, Lanham.

Pardo, Michael S., 2010, "The Gettier Problem and Legal Proof", Legal Theory, vol. 16, no. 1, pp. 37-57.

- 2005, "The Field of Evidence and the Field of Knowledge", Law and Philosophy, vol. 24, no. 4, pp. 321-392.

Pritchard, Duncan, 2010a, "Knowledge and Understanding", en Adrian Haddock, Alan Millar y Duncan Pritchard (comps.), The Nature and Value of Knowledge: Three Investigations, Oxford University Press, Oxford, pp. 1-88.

Pritchard, Duncan, 2010b, "Cognitive Ability and the Extended Cognition Thesis", Synthese, vol. 175, no. 1 sup., pp. 133-15l.

Redondo, María Cristina, 2009, "Sobre la justificación de la decisión judicial", en María Cristina Redondo, José María Sauca y Perfecto Andrés Ibáñez, Estado de derecho y decisiones judiciales, Fundación Coloquio Jurídico Europeo, Madrid, pp. 63-100.

Roberts, Robert C. y W. Jay Wood, 2007, Intellectual Virtues. An Essay in Regulative Epistemology, Clarendon Press, Oxford. 
Sosa, Ernesto, 2007, A Virtue Epistemology, Oxford University Press (Apt Belief and Reflective Knowledge, 1), Oxford.

—_, 1992, Conocimiento y virtud intelectual, trad. A. Zirión et al., Instituto de Investigaciones Filosóficas-UNAM/Fondo de Cultura Económica, México.

—_ 1991, Knowledge in Perspective: Selected Essays in Epistemology, Cambridge University Press, Cambridge.

—_ 1980, "The Raft and the Pyramid: Coherence versus Foundations in the Theory of Knowledge", Midwest Studies in Philosophy, vol. 5, no. 1, pp. 3-25.

Taruffo, Michele, 1992, La prova dei fatti giuridici: nozioni generali, Giuffrè, Milán.

Vaesen, Krist, 2011, "Knowledge without Credit, Exhibit 4: Extended Cognition", Synthese, vol. 181, no. 3, pp. 515-529.

Zagzebski, Linda, 1996, Virtues of the Mind: An Inquiry into the Nature of Virtue and the Ethical Foundations of Knowledge, Cambridge University Press, Cambridge.

Recibido el 18 de noviembre de 2015; revisado el 9 de febrero de 2017; aceptado el 13 de marzo de 2017. 